\title{
Distributed Finite-Time Consensus Control of Second-Order Multiagent Systems Subject to Communication Time Delay
}

\author{
Lingling Fan $\mathbb{D}$ and Chengyan Wu \\ School of Automation, Beijing Information Science and Technology University, Beijing 100192, China \\ Correspondence should be addressed to Lingling Fan; linglingfan@bistu.edu.cn
}

Received 22 October 2021; Revised 3 December 2021; Accepted 4 December 2021; Published 21 December 2021

Academic Editor: Heng Liu

Copyright (c) 2021 Lingling Fan and Chengyan Wu. This is an open access article distributed under the Creative Commons Attribution License, which permits unrestricted use, distribution, and reproduction in any medium, provided the original work is properly cited.

\begin{abstract}
This paper studies the consensus problem of a second-order multiagent system (MAS) with fixed communication delay under the structure of leaderless and leader-following systems. By using graph theory and finite-time control scheme, a distributed control protocol is proposed for each agent to reach consensus in a finite time. In practical application, the time delay of states is unavoidable, and for this, the consensus method is supposed to be extended to solve the time-delay problem. Thus, a finite-time consensus protocol with communication time delay is proposed in this paper. Compared with the general consensus method, the reliability and convergence speed of the system are increased by using the finite-time control. In addition, the protocol is distributed, and all agents have only local interactions. Finally, the effectiveness of the proposed protocol is verified by two numerical simulations.
\end{abstract}

\section{Introduction}

In recent years, the consensus control [1-4] of the multiagent system has attracted great attention, which has been widely applied in the fields of UAV formation [5], intelligent transportation system [6,7], satellite attitude calibration $[8,9]$, aerospace, and other fields. The development of the multiagent system comes from people's research on the overall consensus behavior of biological clusters in nature, such as ants moving, birds flying in clusters, and so on. Consensus problem means that the final state information of each agent in a multiagent system converges to a common value. It is a very meaningful work to design effective control protocols and study how agents can reach agreement within a limited time through cooperation and accomplish tasks that cannot be accomplished by a single agent.

The concept of the multiagent system was first proposed by Minsky [10] in 1986 and later attracted widespread attention. A single entity with the ability of information interaction can be regarded as a single agent. In 2003, Jadbabaie [11] et al. made a specific theoretical analysis of the clustering phenomenon of the model by using the knowledge of graph theory and pointed out the conditions for the system to achieve consensus. Subsequently, Fax and Murray [12] proposed a theoretical research framework for the consensus problem and designed a consensus control algorithm based on the firstorder multiagent system model. Zou et al. [13] studied the distributed consensus tracking problem for heterogeneous switched nonlinear multiagent systems with actuator faults and arbitrary switching signals.

The finite-time consensus (FTC) $[14,15]$ control method has attracted great attention of many researchers in recent years. Long Wang and Feng Xiao [16] analyzed the conditions for the continuous-time multiagent system to achieve consensus based on the finite-time stability theory. In [17], a control strategy based on a velocity-free distributed observer was designed to address the finite-time position consensus tracking control problem for heterogeneous leader-follower multiple AUV systems. In [18], the problem of practical FTC for the second-order MASs with unknown nonlinear dynamics has been studied, in which dynamics are extended to switched nonlinear systems. Li et al. [19] studied 
the finite-time cooperative tracking problem of a class of heterogeneous mixed-order multiagent systems. Zou et al. [20] developed protocols with only state transmissions, by which the consensus can be reached in fixed time.

In practice, due to the communication environment and the communication equipment itself, communication delay inevitably exists in the information transmission between multiple moving agents. Therefore, it is of practical significance to study the consensus of the multiagent system with communication delay [21-23]. Li et al. [24] studied the consensus of leadership following for a first-order multiagent system with noise disturbance and communication delay. Hou et al. [25] studied the consensus problem of a class of general second-order multiagent systems with communication delay by using the relationship between the roots of the characteristic equation and delay parameters. In [22], a distributed algorithm for a second-order multiagent system with nonuniform time delay was proposed to make all agents reach consensus.

Algorithm convergence speed is an important index to evaluate the algorithm performance, especially in some practical complex large-scale systems, which requires the system to be able to converge in a limited time. Considering the possible communication delays in real applications, it is significant to study the time-delay problem with the finitetime control.

Inspired by the above works, we complete the following works. A finite-time consensus problem involved with communication delay is considered in this paper. The communication graph is adopted as a directed connected graph with a fixed topology. Each agent is modelled by a second-order integrator with unknown nonlinear dynamics. In order to verify the effectiveness of the proposed control protocol, leaderless and leaderfollowing systems are selected, respectively, in the numerical simulation. It is proved that the FTC can be achieved by given protocols. The contributions of this paper are summarized as follows:

(1) We use the sign function feedback control method based on partial state information to achieve a finitetime consensus for the MAS with communication time-delay problem when the time delay is under the upper limit. Meanwhile, the protocol is distributed and developed for each agent, and only partial information interaction is required.

(2) Two different structures of leaderless and leaderfollowing multiagent systems have been discussed in this paper. Simulation results indicate that the finitetime consensus can be reached by the given protocols. Compared with the general consensus method which converges asymptotically, the convergence speed of the system is improved effectively by using the finite-time method proposed in this paper.

The outline of this work is as follows. Some preliminary knowledge is given in Section 2. Consensus control protocols proposed for leaderless and leader-following systems to solve the finite-time consensus problem with communication time delay and the proof are given in Section 3. Two examples of numerical simulation showing the effectiveness of the proposed protocol are given in Section 4. The summary of this paper and future research is given in Section 5.

\section{Problem Formulation}

2.1. Graph Theory. Graph theory analysis is an important tool to deal with distributed control problems, which can easily describe the information transmission relationship of a MAS.

In the multiagent system, for simple analysis, each single agent can be regarded as a node, and information can be exchanged between nodes. Then, a graph $G(A)=(V, E, A)$ can be used to describe the interaction between agents; a system of $n$ intelligent agents can be described as a nonempty vertex set of $V(G)=\left\{v_{1}, v_{2}, \ldots, v_{n}\right\} ; E \in V \times V$ represents a collection of nonempty directed edges; $A=\left[a_{i j}\right]_{n \times n} \in R^{n \times n}$ is the weighted adjacency matrix; and $a_{i j}$ represents the weight of an edge in the topology starting at $v_{j}$ and ending at $v_{i}$. Moreover, $a_{i j}>0$ if $\left(v_{j}, v_{i}\right) \in E$; otherwise, $a_{i j}=0$. In addition, $a_{i i}=0$. For example, when the weight is set to 1 , $a_{i j}=1$. The weighted adjacency matrix $B=\operatorname{di} a g\left\{b_{1}, b_{2}, \ldots, b_{n}\right\}$ is defined to describe the information interaction relationship between the leaders and the followers. If the followers can receive the information from the leader, then $b_{i j}>0$, and when there is no information transmission, $b_{i j}=0$.

Define the degree matrix $D=\operatorname{diag}\left\{d_{1}, d_{2}, \cdots, d_{n}\right\}$, where $d_{i}$ represents the out degree of agent $i$, which is equal to the sum of every row of elements in the weighted adjacent matrix $A$. Define the Laplacian matrix $L=D-A$, which is the matrix representation of a topological graph.

2.2. Matrix Theory. Let matrix $A \in R^{m \times n}$ and matrix $B \in R^{p \times q}$; then, the Kronecker product of matrices $A \otimes B$ is a matrix with dimension of $m p \times n q$.

The Kronecker product is described by

$$
A \otimes B=\left(\begin{array}{ccc}
a_{11} B & \ldots & a_{1 n} B \\
\vdots & \ddots & \vdots \\
a_{m 1} B & \cdots & a_{m n} B
\end{array}\right) .
$$

Properties of the Kronecker product are given as follows:

(1) $(A \otimes B)(C \otimes D)=(A C) \otimes(B D)$.

(2) $(A \otimes B)+(A \otimes C)=A \otimes(B+C)$.

(3) $(A B)^{-1}=A^{-1} \otimes B^{-1}$.

(4) $(A \otimes B)^{T}=A^{T} \otimes B^{T}$.

2.3. Notations. Throughout this paper, the following notations are used. $\mathbf{1}_{\mathrm{n}}$ represents an $n$-dimensional row vector with an element of $1 ; I_{n}$ represents the identity matrix of the dimensionality of $n \times n ; \mathbf{0}_{n}$ denotes the zero matrix of 
appropriate dimension; $|\cdot|$ is the modulus of a real number; and $\|\cdot\|$ is the norm of a vector.

\section{System Model and Protocol Design}

3.1. Problem Description. Consider a class of second-order leaderless multiagent systems consisting of $n$ agents. The dynamics of $i$ th agent can be described as

$$
\left\{\begin{array}{l}
\dot{x}_{i}(t)=v_{i}(t) \\
\dot{v}_{i}(t)=f\left(x_{i}(t), v_{i}(t)\right)+u_{i}(t)
\end{array},\right.
$$

where $i=1,2, \cdots, n, \quad x_{i}(t) \in R^{n}$ is the position state, $v_{i}(t) \in R^{n}$ is the velocity state of the $i$ th agent at $t, u_{i}(t) \in R^{n}$ represents the input for agent $i$, and $f\left(x_{i}(t), v_{i}(t)\right)$ is the unknown continuous nonlinear function with uncertainties.

Finite-time consensus is to be achieved when the following equations hold. As time $t$ approaches $T^{*}$, under any given bounded initial state, it satisfies

$$
\begin{aligned}
& \lim _{t \longrightarrow T^{*}}\left\|x_{i}(t)-x_{j}(t)\right\|=0, \\
& \lim _{t \longrightarrow T^{*}}\left\|v_{i}(t)-v_{j}(t)\right\|=0,
\end{aligned}
$$

where $T^{*}$ is finite time.

If we design an effective consensus control protocol to make equations (3) and (4) hold, it can be said that the leader-following multiagent system of the second-order integrator model above can achieve consensus within a finite-time $T^{*}$.

With zero time delay, the general consensus control protocol is given as

$$
u_{i}(t)=-\alpha\left(\sum_{j=1}^{n} L_{i j} x_{j}(t)\right)-\beta\left(\sum_{j=1}^{n} L_{i j} v_{j}(t)\right)
$$

Lemma 1 (see [24]). The control protocol (5) makes the MAS with (5) achieve consensus if and only if $G$ has a directed spanning tree and the following inequalities hold:

$$
\alpha>0, \beta>0, \frac{\beta^{2}}{\alpha}>\max _{i=1,2, \cdots n} \frac{\operatorname{Im}^{2}\left(\lambda_{i}\right)}{\operatorname{Re}\left(\lambda_{i}\right)\left|\lambda_{i}\right|^{2}} .
$$

Choose the appropriate Lyapunov function candidate, and the consensus control protocol of (5) can make the multiagent system described in (2) achieve consensus asymptotically. This was proved in [24].

The following discussion considers the case of communication time delay. Under the structure of leaderless and leader-following systems, effective finite-time consensus protocols were designed for MAS with communication time delay.

\subsection{Finite-Time Consensus Protocol with Communication} Delay for Leaderless MAS. In this section, consider the existence of communication delay in the leaderless system, and the following finite-time control protocol is proposed to solve the consensus problem with time delay.

For the $i$ th agent in the multiagent system, we consider the following control protocol:

$$
u_{i}(t)=-\alpha\left[\sum_{j=1}^{n} L_{i j} x_{j}(t-\tau)+\operatorname{sig}\left(\sum_{j=1}^{n} L_{i j} x_{j}(t-\tau)\right)^{k}\right]-\beta\left[\sum_{j=1}^{n} L_{i j} v_{j}(t-\tau)+\operatorname{sig}\left(\sum_{j=1}^{n} L_{i j} v_{j}(t-\tau)\right)^{k}\right]
$$

where $\tau$ represents the time delay of information transfer from agent $i$ to agent $j$.

We assume that $\tau_{i j}=\tau_{j i}=\tau$, that is, the communication delay between two agents is considered the same.

In (7),

$$
\sum_{j=1}^{n} L_{i j} x_{j}=\sum_{j=1}^{n} a_{i j}\left(x_{i}-x_{j}\right)
$$

Equation (8) represents the relative state information between agent $i$ and its neighbor agent $j$. In equation (7), $\operatorname{sig}(x)^{k}=|x|^{k} \operatorname{sign}(x)$, where $\operatorname{sign}(x)$ is a symbolic function. One has $\alpha>0, \beta>0$.
We select the state of agent 1 to facilitate the calculation and evaluation of the consensus error. Then, we define the position error and velocity error as follows:

$$
\begin{aligned}
& e_{x i}(t)=x_{i}(t)-1_{n-1} x_{1}(t), \\
& e_{v i}(t)=v_{i}(t)-1_{n-1} v_{1}(t),
\end{aligned}
$$

where $i=2,3, \ldots, n$. So, it is easy to know that $e_{x i} \in R^{n-1}$, $e_{v i} \in R^{n-1}$.

$E=\left[e_{x i}, e_{v i}\right]^{T}$ represents the neighborhood error matrix.

Substituting the proposed control protocol (7) into system equation (2), the following error matrix equation can be obtained.

$$
\dot{\mathbf{E}}(t)=\left[\begin{array}{ll}
\mathbf{0}_{n-1} & \mathbf{I}_{n-1} \\
\mathbf{0}_{n-1} & \mathbf{0}_{n-1}
\end{array}\right] \mathbf{E}(t)+\left[\begin{array}{cc}
\mathbf{0}_{n-1} & \mathbf{0}_{n-1} \\
-\alpha \widehat{\mathbf{L}} & -\beta \widehat{\mathbf{L}}
\end{array}\right] \mathbf{E}(t-\tau)
$$




$$
+\left[\begin{array}{cc}
\mathbf{0}_{n-1} & \mathbf{0}_{n-1} \\
-\alpha \mathbf{I}_{n-1} & -\beta \mathbf{I}_{n-1}
\end{array}\right] \operatorname{sig}(\mathbf{E} \overline{\bar{L}}(t-\tau))^{k}+\left[\begin{array}{c}
\mathbf{0}_{(n-1) \times 1} \\
\mathbf{e}_{\mathbf{f}}
\end{array}\right]
$$

where $\quad e_{f}=f_{i}(t)-f_{1}(t), \quad \widehat{L}=L_{22}+1_{n-1} A_{1 n}^{T}, \quad L_{22}=$ $\left[\begin{array}{cccc}d_{2} & -a_{23} & \cdots & -a_{2 n} \\ -a_{32} & d_{3} & \cdots & -a_{3 n} \\ \vdots & \vdots & \ddots & \vdots \\ -a_{n 2} & -a_{n 3} & \cdots & d_{n}\end{array}\right]$,

$\left[\begin{array}{cc}\widehat{L} & 0_{n-1} \\ 0_{n-1} & \widehat{L}\end{array}\right]$. $A_{1 n}=\left[\begin{array}{c}a_{12} \\ a_{13} \\ \vdots \\ a_{1 n}\end{array}\right]$, and $\quad \overline{\widehat{L}}=$
Define $A_{n 1}=\left[\begin{array}{llll}a_{21} & a_{31} & \cdots & a_{n 1}\end{array}\right]^{T}$; then, the Laplacian matrix $L=\left[\begin{array}{cc}d_{1} & -A_{1 n}^{T} \\ -A_{1 n} & L_{22}\end{array}\right]$.

Equation (11) can be written as

$$
\dot{E}(t)=\left(I_{n-1} \otimes B_{1}\right) E(t)+\left(\widehat{L} \otimes B_{2}\right) E(t-\tau)+\left(I_{n-1} \otimes B_{2}\right) \operatorname{sig}(\overline{\widehat{L}} E(t-\tau))^{k}+B_{3},
$$

where $B_{1}=\left[\begin{array}{ll}0 & 1 \\ 0 & 0\end{array}\right], B_{2}=\left[\begin{array}{cc}0 & 0 \\ -\alpha & -\beta\end{array}\right]$, and $B_{3}=\left[\begin{array}{c}0_{(n-1) \times 1} \\ e_{f}\end{array}\right]$.

Apparently, considering the consensus of system (2) translates into studying the convergence of error matrix equation (12).

Lemma 2. Consider the system

$$
\begin{aligned}
\dot{x} & =f(x), \\
f(0) & =0, \\
x & \in R^{n},
\end{aligned}
$$

and there exist a positive definite continuous function $V(x): U \longrightarrow R$, real numbers $c_{1}, c_{2}>0$ and $\alpha \in(0,1)$, and an open neighborhood $U_{0} \subset U$ of the origin such that $\dot{V}(x)+c_{1} V(x)+c_{2} V^{\alpha}(x) \leq 0, x=U_{0} /\{0\}$. Then, $V(x)$ will approach 0 in a finite-time $T^{*}$.
Lamma 3 (see [24]). The Laplacian matrix $L$ has exactly one zero eigenvalue and all the other eigenvalues have positive parts if and only if the directed network has a directed spanning tree.

Assumption 1 In the leaderless multiagent system, assume that the communication topology in the MAS described by equation (2) is represented by a directed graph $G$ and the network topology contains a spanning tree.

Assumption 2 (see [26]). In the leader-following multiagent system, assume that the communication topology in the MAS described by (2) and (24) contains a directed spanning tree in the digraph $\bar{G}=G \cup\{0\}$ with the leader $\{0\}$ as the root node.

Assumption 3 (see [22]). There exist two nonnegative constants $k_{1}$ and $k_{2}$, such that

$$
\left\|f\left(x_{1}(t), x_{2}(t)\right)-f\left(y_{1}(t), y_{2}(t)\right)\right\| \leq k_{1}\left\|x_{1}(t)-y_{1}(t)\right\|+k_{2}\left\|x_{2}(t)-y_{2}(t)\right\|
$$

for any $x_{1}(t), x_{2}(t), y_{1}(t), y_{2}(t) \in R^{n}$.

\subsection{Consensus Analysis}

Theorem 1. Consider the leaderless system with equation (2). Suppose $G$ has a directed spanning tree and that control protocol (7) can make the MAS achieve finite-time consensus in the time-delay case.

Proof. The Lyapunov function is constructed as follows:

$$
V(t)=\frac{1}{2} E^{T}(t) \mathrm{QE}(t),
$$

where $Q=\left[\begin{array}{cc}2 \alpha \beta \widehat{L} & \alpha I_{n-1} \\ \alpha I_{n-1} & \beta I_{n-1}\end{array}\right] \in R^{2(n-1)}$.

When $\beta>0, \lambda_{\min }(L)>\alpha / 2 \beta^{2}$, one has $Q>0$, so that $V(t)$ is positive definite.
Computing the derivative of time for $V(t)$, one has

$$
\dot{V}(t)=E^{T}(t) \mathrm{Q} \dot{E}(t) .
$$

Substituting (12) into (16) yields

$$
\begin{aligned}
\dot{V}(t)= & E^{T}(t) Q\left(I_{n-1} \otimes B_{1}\right) E(t)+E^{T}(t) Q\left(\widehat{L} \otimes B_{2}\right) E(t-\tau) \\
& +E^{T}(t) Q\left(I_{n-1} \otimes B_{2}\right) \operatorname{sig}(E \overline{\hat{L}}(t-\tau))^{k}+E^{T}(t) Q B_{3} \\
= & E^{T}(t) P_{1} E(t)+E^{T}(t) P_{2} E(t-\tau) \\
& +E^{T}(t) P_{3} \operatorname{sig}(\hat{\bar{L}} E(t-\tau))^{k}+E^{T}(t) P_{4},
\end{aligned}
$$

where $\quad P_{1}=\left[\begin{array}{cc}0_{n-1} & 2 \alpha \beta \widehat{L} \\ 0_{n-1} & \alpha I_{n-1}\end{array}\right], \quad P_{2}=\left[\begin{array}{ll}-\alpha^{2} \widehat{L} & -\alpha \beta \widehat{L} \\ -\alpha \beta \widehat{L} & -\beta^{2} \widehat{L}\end{array}\right]$, $P_{3}=\left[\begin{array}{ll}-\alpha^{2} I_{n-1} & -\alpha \beta I_{n-1} \\ -\alpha \beta I_{n-1} & -\beta^{2} I_{n-1}\end{array}\right]$, and $P_{4}=\left[\begin{array}{c}\alpha e_{f} \\ \beta e_{f}\end{array}\right]$. 
Then,

$$
\begin{aligned}
\dot{V}(t)= & {\left[\begin{array}{c}
E(t) \\
E(t-\tau)
\end{array}\right]^{T}\left[\begin{array}{cc}
P_{1} & P_{2} \\
0_{2(n-1)} & 0_{2(n-1)}
\end{array}\right]\left[\begin{array}{c}
E(t) \\
E(t-\tau)
\end{array}\right], } \\
& +\left[\begin{array}{c}
E(t) \\
E(t-\tau)
\end{array}\right]^{T}\left[\begin{array}{cc}
P_{3} & 0_{2(n-1)} \\
0_{2(n-1)} & 0_{2(n-1)}
\end{array}\right] \operatorname{sig}\left(\left[\begin{array}{cc}
0_{2(n-1)} & 0_{2(n-1)} \\
0_{2(n-1)} & \overline{\widehat{L}}
\end{array}\right]\left[\begin{array}{c}
E(t) \\
E(t-\tau)
\end{array}\right]\right)^{k}, \\
& +\left[\begin{array}{c}
E(t) \\
E(t-\tau)
\end{array}\right]^{T}\left[\begin{array}{c}
\alpha e_{f}+\beta e_{f} \\
0_{2(n-1) \times 1}
\end{array}\right]=-z^{T} M_{1} z-z^{T} M_{2} \operatorname{sig}(z)^{k}-z^{T} M_{3},
\end{aligned}
$$

where $z=\left[\begin{array}{c}E(t) \\ E(t-\tau)\end{array}\right], \quad M_{1}=\left[\begin{array}{cc}-P_{1} & -P_{2} \\ 0_{2(n-1)} & 0_{2(n-1)}\end{array}\right], \quad M_{2}=$ $\left[\begin{array}{cc}-P_{3} \overline{\widehat{L}}^{k} & 0_{2(n-1)} \\ 0_{2(n-1)} & 0_{2(n-1)}\end{array}\right]$, and $M_{3}=\left[\begin{array}{c}-\left(\alpha e_{f}+\beta e_{f}\right) \\ 0_{2(n-1) \times 1}\end{array}\right]$.

From (18), one has

$$
\dot{V}(t) \leq-\lambda_{\text {min }}\left(M_{1}\right)\|z\|^{2}-\lambda_{\text {min }}\left(M_{2}\right)\|z\|^{k+1}-\left\|M_{3}\right\|\|z\|=-m_{1}\|z\|^{2}-m_{2}\|z\|^{k+1}-\left\|M_{3}\right\|\|z\|,
$$

where $m_{1}=\lambda_{\min }\left(M_{1}\right)$ and $m_{2}=\lambda_{\min }\left(M_{2}\right)$.

Also, it can be known from Assumption 3 that

$$
\begin{aligned}
\left\|M_{3}\right\| & =|\alpha+\beta|\left\|e_{f}\right\|=|\alpha+\beta|\left\|f\left(x_{i}(t), v_{i}(t)\right)-f\left(x_{1}(t), v_{1}(t)\right)\right\|, \\
& \leq|\alpha+\beta|\left(k_{1}\left\|x_{i}(t)-x_{1}(t)\right\|+k_{2}\left\|v_{i}(t)-v_{1}(t)\right\|\right), \\
& =|\alpha+\beta|\left(k_{1}\left\|e_{x i}\right\|+k_{2}\left\|e_{v i}\right\|\right), \\
& =\left[\left(k_{1}|\alpha+\beta|+k_{2}|\alpha+\beta|\right) 1_{1 \times 2(n-1)} 0_{1 \times 2(n-1)}\right]\left[\begin{array}{c}
E(t) \\
E(t-\tau)
\end{array}\right], \\
& \leq|\alpha+\beta| \sqrt{k_{1}^{2}+k_{2}^{2}}\|z\| .
\end{aligned}
$$

Then, substituting (20) into (19), one has

$$
\dot{V}(t) \leq-\left(m_{1}+|\alpha+\beta| \sqrt{k_{1}^{2}+k_{2}^{2}}\right)\|z\|^{2}-m_{2}\|z\|^{k+1} .
$$

According to equation (15),

$$
V(t)=\frac{1}{2}\left[\begin{array}{c}
E(t) \\
E(t-\tau)
\end{array}\right]^{T}\left[\begin{array}{cc}
Q & 0_{2(n-1)} \\
0_{2(n-1)} & 0_{2(n-1)}
\end{array}\right]\left[\begin{array}{c}
E(t) \\
E(t-\tau)
\end{array}\right] \leq \lambda_{\max }(\bar{Q})\|z\|^{2}=m_{0}\|z\|^{2},
$$

where $\bar{Q}=\left[\begin{array}{cc}1 / 2 Q & 0_{2(n-1)} \\ 0_{2(n-1)} & 0_{2(n-1)}\end{array}\right], m_{0}=\lambda_{\max }(\bar{Q})=\beta+2 \alpha \beta \lambda_{\max }$ $\dot{V}(t) \leq-\frac{\left(m_{1}+|\alpha+\beta| \sqrt{k_{1}^{2}+k_{2}^{2}}\right)}{m_{0}} V(t)-\frac{m_{2}}{m_{0}{ }^{1+k / 2}} V^{1+k / 2}(t)$. $(\widehat{L}) / 4+\sqrt{\left(\beta / 2+\alpha \beta \lambda_{\max }(\widehat{L})\right)^{2}-2 \alpha \beta^{2} \lambda_{\max }(\widehat{L})+\alpha^{2}} / 2$, and $\lambda_{\max }(\widehat{L})$ is the minimum eigenvalue of the matrix $\widehat{L}$. Substituting (22) into (21) yields

Thus, according to Lemma 2 , the system converges in a finite time. This completes the proof. 
3.4. Finite-Time Consensus Protocol with Communication Delay for Leader-Following MAS. In the previous section, we analyzed the consensus problem for leaderless system, and the final consensus state converges to some function related to the agent's initial state. In some practical applications, all agents are desired to converge to a specified value eventually. Then, we add a leader to the system to solve the problem of consensus tracking in this section.
The dynamics of the leader agent are described by

$$
\left\{\begin{array}{l}
\dot{x}_{0}(t)=v_{0}(t) \\
\dot{v}_{0}(t)=f\left(x_{0}(t), v_{0}(t)\right)
\end{array},\right.
$$

and the followers are described as in equation (2).

In this case, for the leader-following system, the distributed finite-time consensus control protocol is proposed:

$$
\begin{aligned}
u_{i}(t)= & -\alpha\left[\sum_{j=1}^{n} L_{i j} x_{j}(t-\tau)+\operatorname{sig}\left(\sum_{j=1}^{n} L_{i j} x_{j}(t-\tau)\right)^{k}\right]-\beta\left[\sum_{j=1}^{n} L_{i j} v_{j}(t-\tau)+\operatorname{sig}\left(\sum_{j=1}^{n} L_{i j} v_{j}(t-\tau)\right)^{k}\right] \\
& -b_{i}\left[x_{i}(t-\tau)-x_{0}(t-\tau)+v_{i}(t-\tau)-v_{0}(t-\tau)\right],
\end{aligned}
$$

where $b_{i}$ is the diagonal element in the adjacency matrix $B=\left[b_{1}, b_{2}, \ldots, b_{n}\right]^{T}$.

Theorem 2. Consider the leader-following system with equations (2) and (24), and control protocol (25) can make the MAS achieve finite-time consensus in the time-delay case.

Proof. Define the position error and velocity error as follows:

$$
\begin{aligned}
& \delta_{x i}(t)=x_{i}(t)-1_{n} x_{0}(t), \\
& \delta_{v i}(t)=v_{i}(t)-1_{n} v_{0}(t),
\end{aligned}
$$

where $i=1,2, \ldots, n, e_{x i} \in R^{n}, e_{v i} \in R^{n}$.
Defining $\delta=\left[\delta_{x i}, \delta_{v i}\right]^{T}$ and taking the derivative of $\delta$, one has

$$
\begin{aligned}
\dot{\delta}(t)= & {\left[\begin{array}{ll}
0_{n} & I_{n} \\
0_{n} & 0_{n}
\end{array}\right] \delta(t)+\left[\begin{array}{cc}
0_{n} & 0_{n} \\
-\alpha L-B & -\beta L-B
\end{array}\right] \delta(t-\tau) } \\
& +\left[\begin{array}{cc}
0_{n} & 0_{n} \\
-\alpha I_{n} & -\beta I_{n}
\end{array}\right] \operatorname{sig}(\bar{L} \delta(t-\tau))^{k}+\left[\begin{array}{c}
0_{n \times 1} \\
\delta_{f}
\end{array}\right],
\end{aligned}
$$

where $\bar{L}=\left[\begin{array}{cc}L & 0_{n} \\ 0_{n} & L\end{array}\right]$ and $\delta_{f}=f_{i}(t)-f_{0}(t)$.

Equation (27) can be written as

$$
\dot{\delta}(t)=\left(I_{n} \otimes B_{1}\right) \delta(t)+\left(L \otimes \mathrm{B}_{2}+\mathrm{B} \otimes B_{0}\right) \delta(t-\tau)+\left(I_{n} \otimes \mathrm{B}_{2}\right) \operatorname{sig}(\bar{L} \delta(t-\tau))^{k}+\mathrm{B}_{3},
$$

where $B_{0}=\left[\begin{array}{cc}0 & 0 \\ -1 & -1\end{array}\right], B_{1}=\left[\begin{array}{ll}0 & 1 \\ 0 & 0\end{array}\right], B_{2}=\left[\begin{array}{cc}0 & 0 \\ -\alpha & -\beta\end{array}\right]$, and $B_{3}=\left[\begin{array}{c}0_{n \times 1} \\ \delta_{f}\end{array}\right]$, which are the same as in (12).

Note that (12) and (28) have the same structure, and the rest is similar to the proof of Theorem 1 described in Section 3.3; we can also select the same Lyapunov function to prove that the system can converge in a finite time. Thus, it is omitted here. This completes the proof.

\section{Numerical Simulation}

Example 1. Consider a second-order leaderless MAS described by equation (2), consisting of $n=6$ follower agents. The communication topology is shown in Figure 1, whose weights are taken as follows.

From the graph theory knowledge, we know that

$\mathbf{A}=\left[\begin{array}{llllll}0 & 0 & 0 & 0 & 0 & 0 \\ 1 & 0 & 0 & 0 & 0 & 0 \\ 2 & 0 & 0 & 0 & 0 & 0 \\ 0 & 0 & 1 & 0 & 0 & 0 \\ 0 & 0 & 3 & 1 & 0 & 0 \\ 0 & 0 & 0 & 2 & 0 & 0\end{array}\right]$,

$$
\mathbf{L}=\left[\begin{array}{cccccc}
0 & 0 & 0 & 0 & 0 & 0 \\
-1 & 1 & 0 & 0 & 0 & 0 \\
-2 & 0 & 2 & 0 & 0 & 0 \\
0 & 0 & -1 & 1 & 0 & 0 \\
0 & 0 & -3 & -1 & 4 & 0 \\
0 & 0 & 0 & -2 & 0 & 2
\end{array}\right]
$$




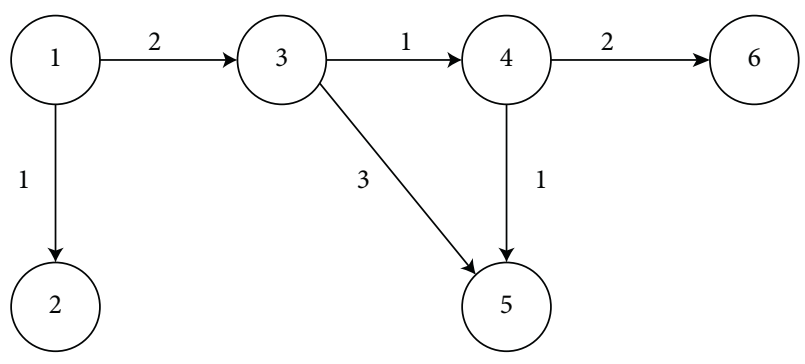

Figure 1: Topology of the graph G.

The initial states of each agent in the multiagent system

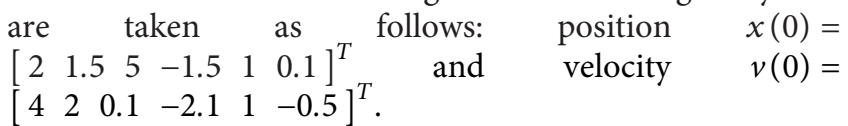

The nonlinear functions are selected as follows: $f(t)=-\sin (x)-0.25 \sin (v)+1.5 \cos (2.5 t)$.

Choose the simulation duration as $T=20 \mathrm{~s}$. The values of the parameters in the control protocol are taken as follows: $\alpha=1.5, \beta=1.5, k=0.8$, and $\tau=0.06$.

Figure 2 shows the simulation results when finite-time control protocol (7) is adopted.

It can be observed that the position and the velocity of the agents to reach a consensus takes about $9 \mathrm{~s}$, which indicates that the consensus protocol is effective and the finitetime consensus for the time-delay case is achieved.

The state error of the leaderless system is defined as in (9) and (10).

Figure 3 shows the convergence of the state errors. It can be seen that the position and the velocity error converge to zero in a finite time.

Within the same context, general consensus protocol with communication time delay based on (5) could be designed as

$$
u_{i}(t)=-\alpha\left(\sum_{j=1}^{n} L_{i j} x_{i}(t-\tau)\right)-\beta\left(\sum_{j=1}^{n} L_{i j} v_{i}(t-\tau)\right) .
$$

The consensus control protocol of (30) can make the leaderless multiagent system described in (2) achieve consensus asymptotically for the time-delay case. This was proved in [17].

Figure 4 shows the position and velocity state error when consensus protocol (30) is adopted.

It can be observed that the state of the agents can achieve consensus around $12 \mathrm{~s}$, which is about $3 \mathrm{~s}$ slower than adopted protocol (7) proposed in Section 3.2.

Compared with the simulation result in Figure 3, it can be seen that the finite-time control protocol designed in this paper can accelerate the convergence speed of the system.

Define the MAE of the agents over the time interval $[0, N]$.

$$
\begin{aligned}
& M A E_{-} x_{i}=\frac{1}{N} \sum_{k=1}^{N}\left|x_{i}-x_{0}\right|, \\
& M A E_{-} v_{i}=\frac{1}{N} \sum_{k=1}^{N}\left|v_{i}-v_{0}\right|,
\end{aligned}
$$

where $N$ represents the running time of the system in the simulation. For example, when the simulation duration is taken as $T$ and the time interval is taken as $T_{s}$, then we have $N=T / T_{s}+1$.

By calculating the mean absolute error (MAE) of the state of the agents, the performance of the algorithm with different consensus protocols (7) and (30) can be evaluated numerically.

According to Table 1, it can be intuitively seen that when finite-time protocol (7) is adopted, the MAE of the multiagent system is smaller, and the state error can converge to zero in a faster time.

Example 2. Consider a leader-following MAS described by equations (2) and (24). The communication topology is shown in Figure 5.

Choose the initial states of each agent in the multiagent system as follows: $x_{1}(0)=\left[\begin{array}{lll}0.2 & 0.4 & 0.5\end{array}\right]^{T}, \quad x_{2}(0)=$

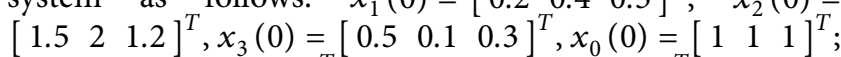
$v_{1}(0)=\left[\begin{array}{lll}-0.2 & 0.4 & 1\end{array}\right]^{T}, \quad v_{2}(0)=\left[\begin{array}{lll}1 & 0.2 & 0.8\end{array}\right]^{T}, \quad v_{3}(0)=$ $\left[\begin{array}{lll}0.4 & 0.5 & 1.2\end{array}\right]^{T}$, and $v_{0}(0)=\left[\begin{array}{lll}0.5 & 1 & 1\end{array}\right]^{T}$. The state of each agent has a three-order component.

The nonlinear functions are selected as

$$
f\left(t, x_{i}, v_{i}\right)=0.5 *\left(\begin{array}{c}
9 v_{i 2}(t)-\frac{18}{7} v_{i 1}(t)+\frac{24}{7}\left(\left|v_{i 1}(t)+1\right|-\left|v_{i 1}(t)-1\right|\right) \\
v_{i 1}(t)-v_{i 2}(t)+v_{i 3}(t) \\
-\frac{100}{7} v_{i 2}(t)
\end{array}\right)
$$

Similar to Example 1, choose the simulation duration as $T=20 \mathrm{~s}$, and the values of the parameters are taken as follows: $\alpha=1.5, \beta=1.5, k=0.8$, and $\tau=0.06$.
Figure 6(a) shows the position of the agents when finitetime control protocol (25) is adopted. Figure 6(b) shows the convergence of the position error. The position error converges to zero in a finite time. 

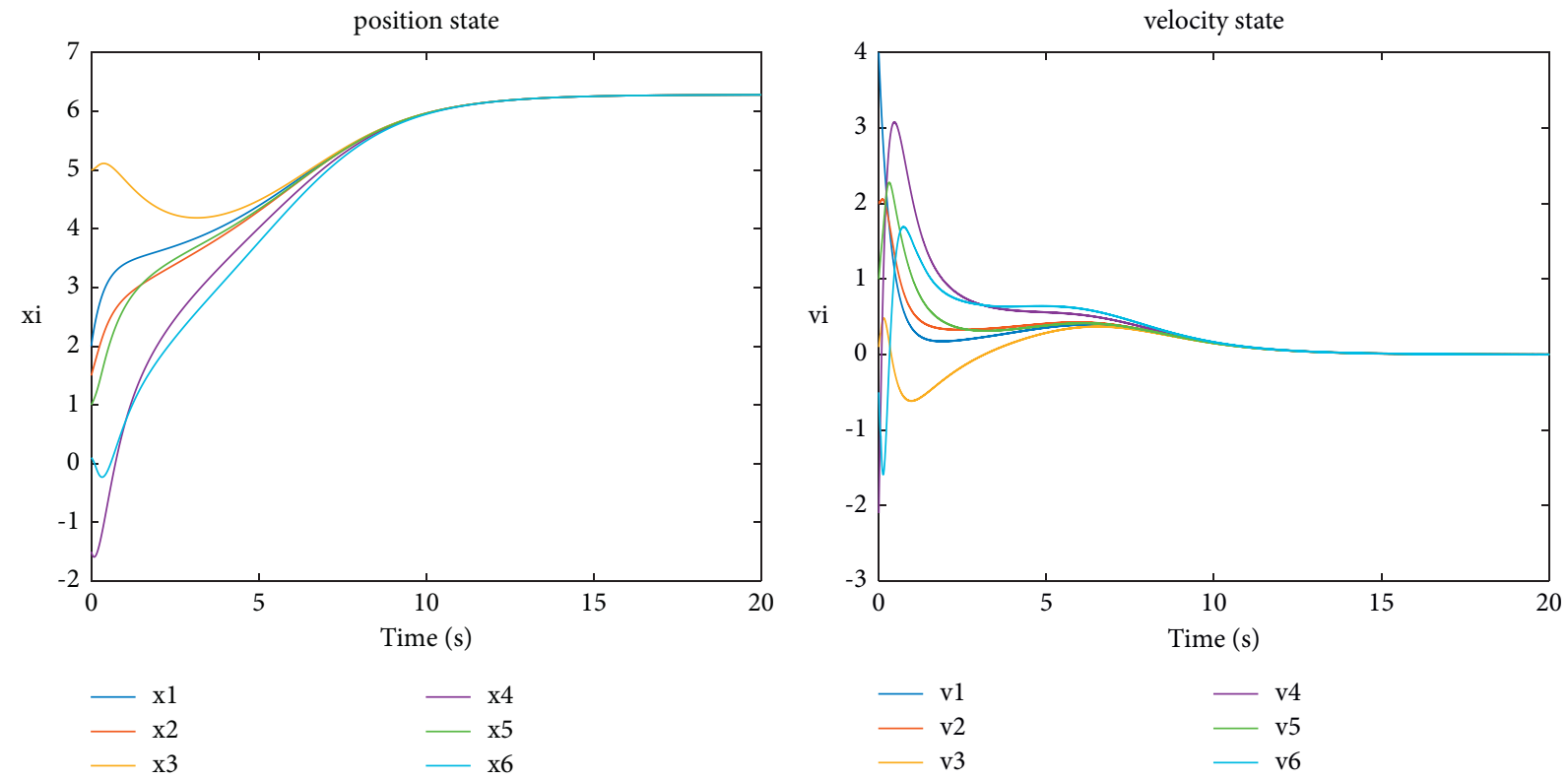

(a)

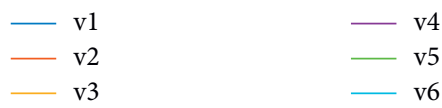

(b)

FIgUre 2: The state of each agent. (a) Position state of each agent. (b) Velocity state of each agent.
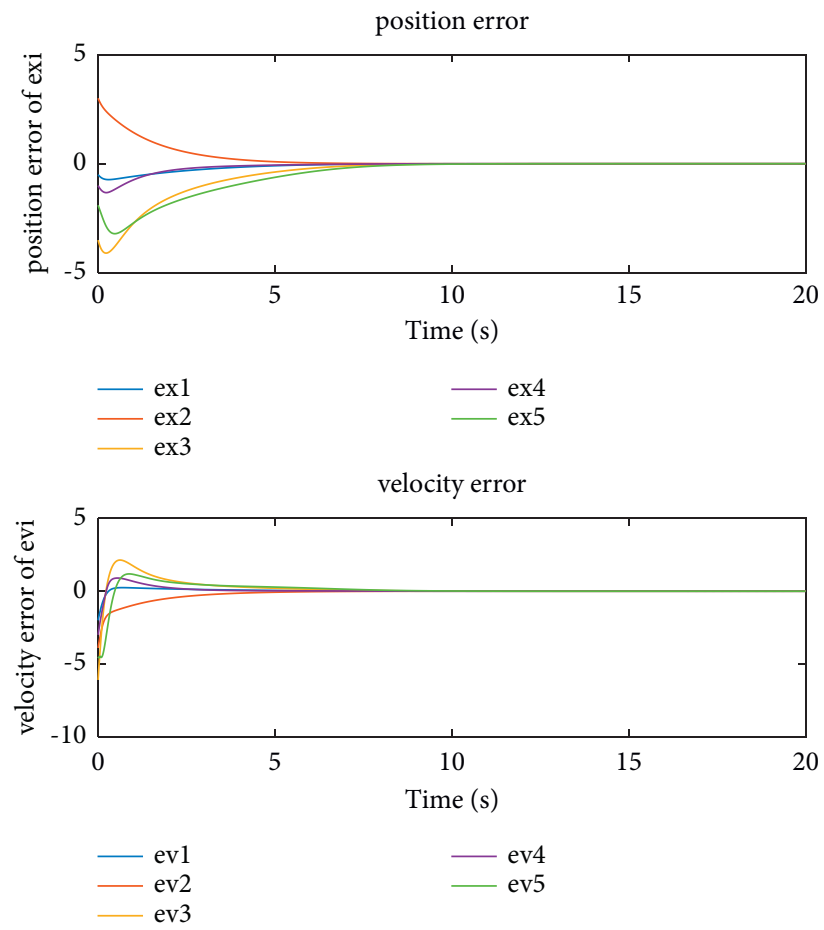

Figure 3: Position error and velocity error. 

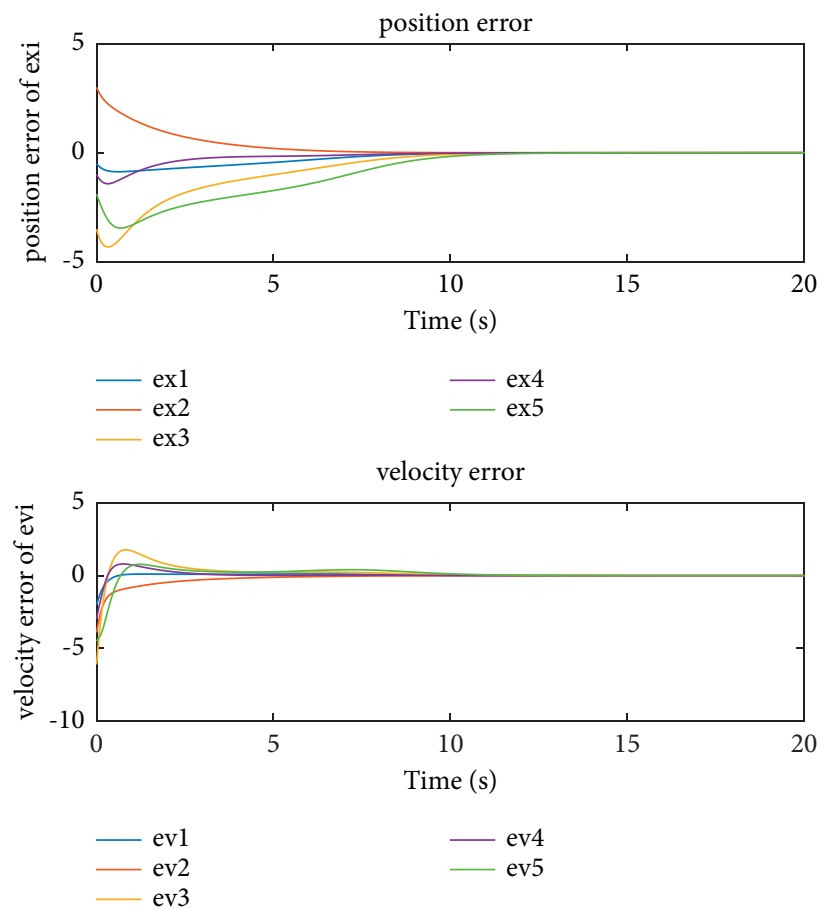

FIgure 4: Position and velocity state error.

TABLE 1: The mean absolute error of the two methods.

\begin{tabular}{lcc}
\hline Methods & MAE $\__{i}$ & MAE $\_v_{i}$ \\
\hline With finite-time protocol (7) & 0.9127 & 0.4984 \\
With general protocol (30) & 1.6477 & 0.5828 \\
\hline
\end{tabular}

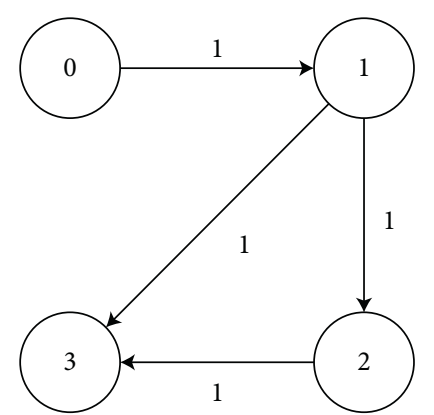

Figure 5: Topology of the graph G.

It can be observed that the position of the agents to reach a consensus takes about $8 \mathrm{~s}$, which indicates that the consensus protocol is effective for the leader-following system with time delay.
Under the structure of the leader-following system, general consensus protocol with communication time delay based on (5) could be designed as

$$
u_{i}(t)=-\alpha\left[\sum_{j=1}^{n} L_{i j} x_{j}(t-\tau)\right]-\beta\left[\sum_{j=1}^{n} L_{i j} v_{j}(t-\tau)\right]-b_{i}\left[x_{i}(t-\tau)-x_{0}(t-\tau)+v_{i}(t-\tau)-v_{0}(t-\tau)\right]
$$



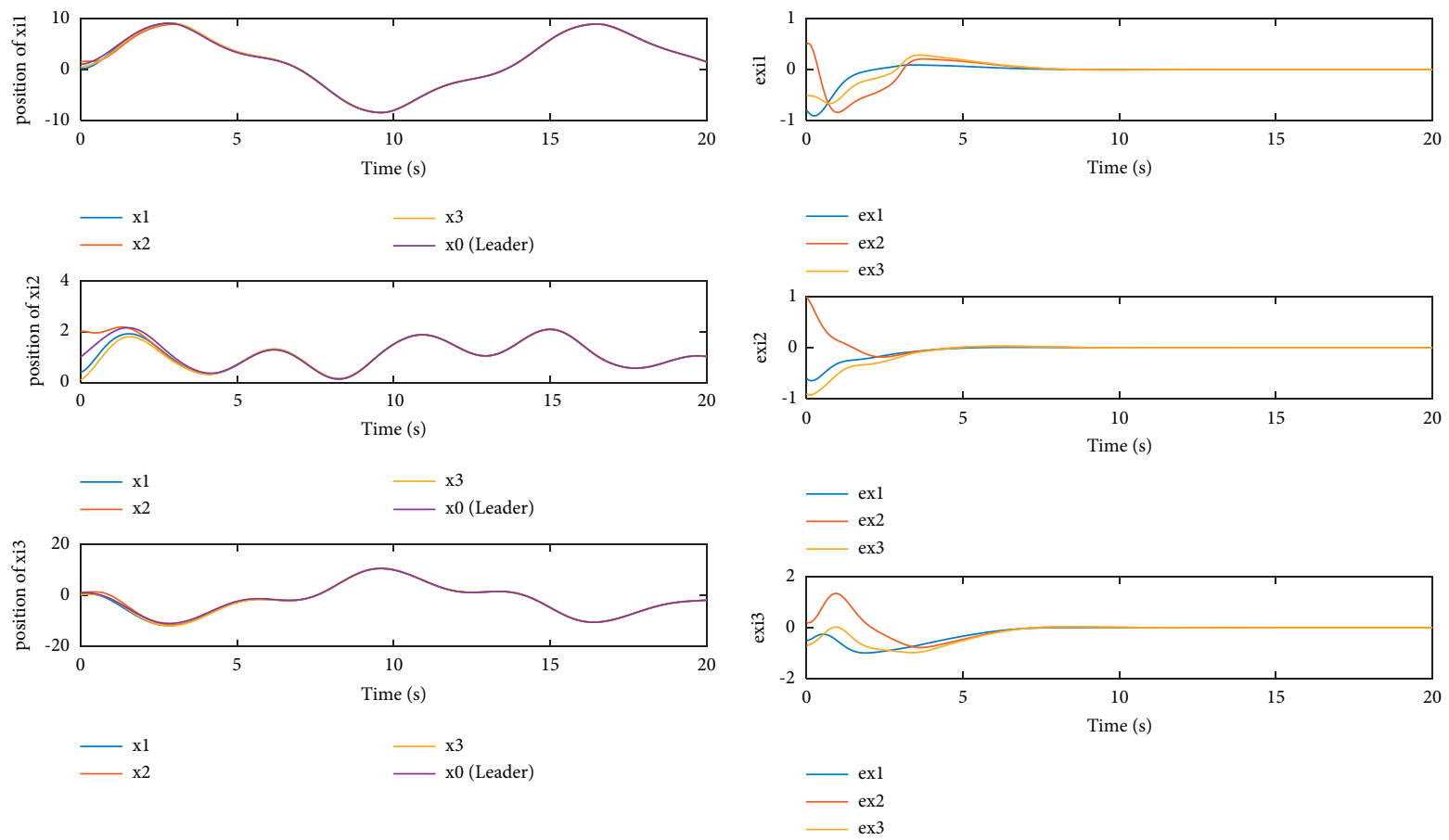

(a)

(b)

Figure 6: Position state and position error. (a) Position state. (b) Position error.
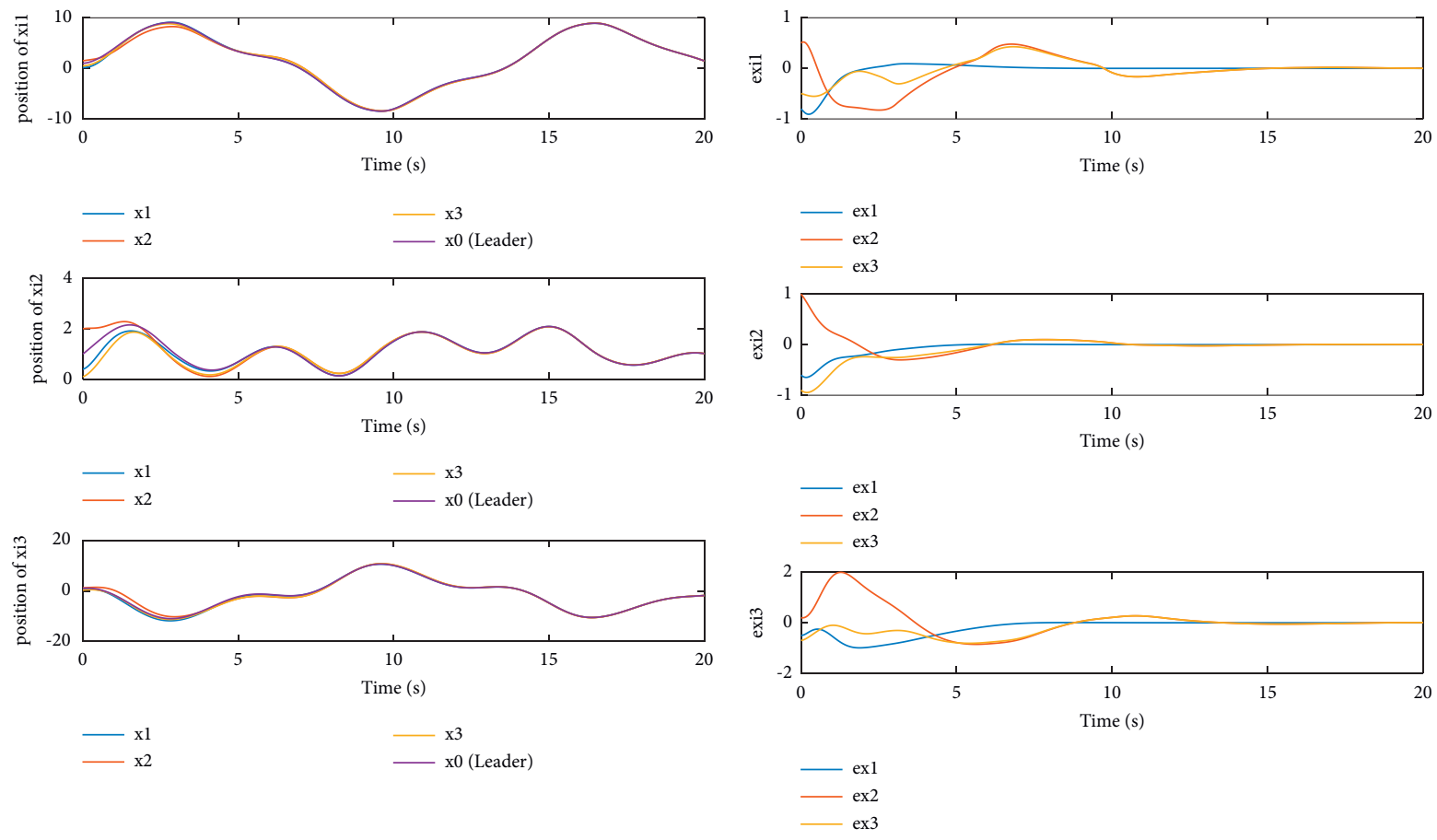

(a)

(b)

Figure 7: Position state and position error. (a) Position state. (b) Position error. 
TABLE 2: The mean absolute error of the two methods.

\begin{tabular}{|c|c|c|c|c|c|c|}
\hline \multirow{2}{*}{ Methods } & \multicolumn{6}{|c|}{ MAE } \\
\hline & $x_{i 1}$ & $x_{i 2}$ & $x_{i 3}$ & $v_{i 1}$ & $v_{i 2}$ & $v_{i 3}$ \\
\hline With finite-time protocol (25) & 0.2345 & 0.1249 & 0.4507 & 0.2176 & 0.0792 & 0.3968 \\
\hline With general protocol (33) & 0.3755 & 0.1897 & 0.5314 & 0.2434 & 0.1099 & 0.4289 \\
\hline
\end{tabular}

Figures 7(a) and 7(b) show the position state and error when consensus protocol (33) is adopted. Compared with Figures 6(a) and 6(b), note that the state of the agents can achieve consensus around $15 \mathrm{~s}$, which is about $7 \mathrm{~s}$ slower than adopted protocol (25).

The simulation results of the agent here only give the position state and position error, and the velocity is similar, which is not given here.

We calculate the mean absolute error (MAE) in this case.

Table 2 shows the MAE of the three-order components of the agent's position state and velocity state. When finitetime protocol (25) is adopted, the MAE is smaller and the state error can converge to zero in a faster time.

\section{Conclusions}

In this paper, the consensus problem with fixed time delay is studied, and finite-time control protocols for the time-delay case are proposed. Two different structures of leaderless and leader-following multiagent systems have been discussed in this paper. Simulation result shows that the position and speed states of all agents can converge to the same in a fast time. Compared with the general consensus protocol, the proposed method can accelerate the convergence speed of the system and make the system more reliable. However, there are still many problems that remain to be solved, such as how to calculate the maximum delay limit of consensus. In addition, the fixed time delay is considered in this paper, and the future research on time-varying delay also presents greater challenges.

\section{Data Availability}

All datasets generated for this study are included within the article.

\section{Conflicts of Interest}

The authors declare that there are no conflicts of interest regarding the publication of this paper.

\section{Acknowledgments}

This study was supported by the National Natural Science Foundation (NNSF) of China (grant no. 61803036), Science Research Program of BISTU (2121YJPY214), and Scientific Research Common Program of Beijing Municipal Commission of Education (KM201911232015).

\section{References}

[1] Z. Li, W. Ren, X. Liu, and L. Xie, "Distributed consensus of linear multi-agent systems with adaptive dynamic protocols," Automatica, vol. 49, no. 7, pp. 1986-1995, 2013.
[2] H. Li, X. Liao, and G. Chen, "Leader-following finite-time consensus in second-order multi-agent networks with nonlinear dynamics," International Journal of Control, Automation and Systems, vol. 11, no. 2, pp. 422-426, 2013.

[3] F. Sun and Z.-H. Guan, "Finite-time consensus for leaderfollowing second-order multi-agent system," International Journal of Systems Science, vol. 44, no. 4, pp. 727-738, 2013.

[4] Y. Wu, J. Hu, L. Xiang, Q. Liang, and K. Shi, "Finite-time output regulation of linear heterogeneous multi-agent systems," IEEE Transactions on Circuits and Systems II: Express Briefs, 2021, In press.

[5] J. Wang, Z. Zhou, C. Wang, and Z. Ding, "Cascade structure predictive observer design for consensus control with applications to UAVs formation flying," Automatica, vol. 121, 2020.

[6] H. Zhang, X. Liu, H. Ji, Z. Hou, and L. Fan, "Multi-agentbased data-driven distributed adaptive cooperative control in urban traffic signal timing," Energies, vol. 12, no. 7, p. 1402, 2019.

[7] D. R. Aleko and D. Soufiene, "An efficient adaptive traffic light control system for urban road traffic congestion reduction in smart cities," Information, vol. 11, no. 2, p. 119, 2020.

[8] M. Kiani, S. H. Pourtakdoust, and A. A. Sheikhy, "Consistent calibration of magnetometers for nonlinear attitude determination,” Measurement, vol. 73, pp. 180-190, 2015.

[9] R. Haghighi and C. K. Pang, "Robust concurrent attitudeposition control of a swarm of underactuated Nanosatellites," IEEE Transactions on Control Systems Technology, vol. 26, no. 1, pp. 77-88, 2018.

[10] M. L. Minsky, The Society of Mind, pp. 371-396, Simon \& Schuster, New York, NY, USA, 1988.

[11] A. Jadbabaie, J. Jie Lin, and A. S. Morse, "Coordination of groups of mobile autonomous agents using nearest neighbor rules," IEEE Transactions on Automatic Control, vol. 48, no. 6, pp. 988-1001, 2003.

[12] J. A. Fax and R. M. Murray, "Information flow and cooperative control of vehicle formations," IEEE Transactions on Automatic Control, vol. 49, no. 9, pp. 1465-1476, 2004.

[13] W. Zou, C. K. Ahn, and Z. Xiang, "Fuzzy-approximationbased distributed fault-tolerant consensus for heterogeneous switched nonlinear multiagent systems," IEEE Transactions on Fuzzy Systems, vol. 29, no. 10, pp. 2916-2925, 2021.

[14] P. Li and J. Hu, "An ADMM based distributed finite-time algorithm for economic dispatch problems," IEEE Access, vol. 6, pp. 30969-30976, 2018.

[15] H. Du, G. Wen, G. Chen, J. Cao, and F. E. Alsaadi, "A distributed finite-time consensus algorithm for higher-order leaderless and leader-following multiagent systems," IEEE Transactions on Systems, Man, and Cybernetics: Systems, vol. 47, no. 7, pp. 1625-1634, 2017.

[16] L. Long Wang and F. Feng Xiao, "Finite-time consensus problems for networks of dynamic agents," IEEE Transactions on Automatic Control, vol. 55, no. 4, pp. 950-955, 2010.

[17] B. Chen, J. Hu, Y. Zhao, and B. Kumar Ghosh, "Finite-time velocity-free observer-based consensus tracking for 
heterogeneous uncertain AUVs via adaptive sliding mode control," Ocean Engineering, vol. 237, Article ID 109565, 2021.

[18] W. Zou, "Finite-time consensus of second-order switched nonlinear multi-agent systems," IEEE transactions on neural networks and learning systems, vol. 31, no. 5, pp. 1757-1762, 2019.

[19] X. Li, P. Shi, Y. Wang, and S. Wang, "Cooperative tracking control of heterogeneous mixed-order multiagent systems with higher-order nonlinear dynamics," IEEE Transactions on Cybernetics, 2020, In press.

[20] W. Zou, K. Qian, and Z. Xiang, "Fixed-time consensus for a class of heterogeneous nonlinear multiagent systems," IEEE Transactions on Circuits and Systems II: Express Briefs, vol. 67, no. 7, pp. 1279-1283, 2019.

[21] J. $\mathrm{Hu}$, "On robust consensus of multi-agent systems with communication delays," Kybernetika, vol. 45, no. 5, pp. 768-784, 2009.

[22] Y. Huang, Y. Li, and W. Hu, "Distributed rotating formation control of second-order leader-following multi-agent systems with nonuniform delays," Journal of the Franklin Institute, vol. 356, no. 5, pp. 3090-3101, 2019.

[23] S. Li, X. Peng, Y. Tang, and Y. Shi, "Finite-time synchronization of time-delayed neural networks with unknown parameters via adaptive control," Neurocomputing, vol. 308, no. 9, pp. 65-74, 2018.

[24] W. Li, Z. Chen, and Z. Liu, "Leader-following formation control for second-order multiagent systems with timevarying delay and nonlinear dynamics," Nonlinear Dynamics, vol. 72 , no. 4, pp. 803-812, 2013.

[25] W. Hou, M. Fu, H. Zhang, and Z. Wu, "Consensus conditions for general second-order multi-agent systems with communication delay," Automatica, vol. 75, no. 6, pp. 293-298, 2017.

[26] Y. Wang, J. Cao, H. Wang, and F. E. Alsaadi, "Event-triggered consensus of multi-agent systems with nonlinear dynamics and communication delay," Physica A: Statistical Mechanics and Its Applications, vol. 522, pp. 147-157, 2019. 